\title{
Product quality pointers for small lots production: A new driver for Quality Management Systems
}

\author{
Matteo M. Savino \\ Department of Engineering, University of Sannio - Piazza Roma, 21, 82100, \\ Benevento, Italy - matteo.savino@unisannio.it \\ Stefano Apolloni \\ Department of Design and Industrial Engineering, University Federico II of \\ Naples, P.le V. Tecchio, 80 - 80125, Naples, Italy - apolloni@unina.it

\section{Yacine Ouzrout} \\ PRISMa-Lyon2 Laboratory, Université Lyon 2 - 160 Bd de l'université 69676 \\ Bron Cedex, France - yacine.ouzrout@univ-lyon2.fr
}

Quality Management Systems (QMS), can be a strategic tool in order to rationalize and improve administrative and industrial management because it allows - in a logic of continuous improvement - to identify a set of quality pointers able to monitor the processes. Recent renewals of QMS have involved a redefinition of pointers system, through the restyling of the existing procedures and the introduction of new processes, having the goal to guarantee an effective control of the processes and therefore to turn out the best one in terms of satisfaction of user's requirement.

The aim of this work is to develop and apply a QMS methodology able to point out a set of pointers finalized to measure objectively the obtained improvements or to define the lacks of a certain production system, considering those particular production systems in which personalization of the product is very emphasized.

The methodology has been applied to the production process of a SME producing motorcaravans and motorhomes, characterized by a high level of product personalization, that often makes it to become a single-type production. The improvements obtained through the implementation of our approach has been mainly related to a decreasing of non conformities (NC) in production of new types up to $25 \%$ and, as a further result, the QMS implemented with our approach has given the same 
performance with new products introduction and at the change of the production types.

Keywords: Quality Management, Small Lot Production, Quality Pointers

\section{Biographical notes:}

Matteo Savino is confirmed researcher and professor of Industrial Operation and Industrial Management at University of Sannio in Benevento. He has got in 1999 a Ph.D. in Industrial Plants at University Federico II of Naples. Actually his research interests are mainly related to ERP management and Supply Chain problems, Quality aspects related to industrial production and Scheduling of Production systems. He has been involved in several research and educational projects, as ERESPLAN and ERPMAN related to configuration and maintenance of ERP, and has been an external evaluator for some projects of the European Commission.

Stefano Apolloni is lecturer at the "Federico II" University of Naples. He received a Phd in industrial engineering from the same university in 2002. His current research interests are health safety, risk environmental, industrial management and process simulation

Yacine Ouzrout obtained his $\mathrm{PhD}$ in Computer Science from the National Institute of Technology of Lyon in France. He is currently an associate professor at the Lyon 2 University. His research interests include simulation, information systems for the Supply Chain, and Product Lifecycle Management. 


\section{Introduction}

The actual industrial scenery is characterized by an ever growing competition which involves every factory aspects: strategies, relations with customers and suppliers, benchmarking activities, skills of factory staff and the utilization of their potential. The international competition imposes to the SMEs to improve products and service offered to the clients, also in case of high personalised products or for small lots production (Oakland et al. - 2006, Reid - 2006) .

It's well known that quality improvement is accomplished through the determination of specific "Quality Pointers" (Mishra et al - 2006). They represent the "check" element which allows to measure factory performances. After six sigma method, applied mostly inside big firms and with high volumes of products, actually several "process pointers" are used, considered as the "final numeric result" of a certain process (McAdam et al - 2004). Choosing these pointers, with related priorities, is a very delicate moment to value the trend of the system and to decide every action to be taken. Approaches which improve these verifying phase are several (Laosirihongthong - 2004), but it's very difficult to individuate an integrated approach which is able to solve problems of several productive processes, made to realize different products, some of them totally new or totally renewed. Moreover the possibility of a sound decreasing of defect percentage is strictly related to the presence of a method able to measure the quality performances of a certain productive system and, at the same time, an objective system able to define the priority of actions (Amrik et al. - 1998, Louis et al. - 2001, Hothard et al. - 1988).

\section{Non Conformity classification}

As well known a Non-Conformity (NC) is a mistake that is find in some phases of a production process and/or on the finished product, or something that did not go as planned and, usually, it is characterised by a weight representing its importance referring to the whole process (Lillrank -2006).

Usually in QMS the classification of NC is made by two categories Dale et al. - 1998, Calligaro - 2002):

- Generic NC (GNC): related to every rejected items that satisfy product requirements. In our opinion GNC are physiological and 
expectable and it depends on the abilities and competencies of the workers; it means that normally GNC is accepted by customer

- Critical NC (CNC): CNC are every NC having a high frequency of happening and/or those that are perceived by the customer and generate complains or reject the item.

Afterwards, with respect to the aim of this work, we have subdivided the $\mathrm{NC}$ in two subcategories:

- $\mathrm{NC}$ of the products belonging to an offer range previewed by the factory

- NC belonging to products completely personalized

\section{Efficiency classes definition $-\mathbf{I}_{\text {REM }}$ and $\mathbf{I}_{\text {REP }}$}

Moreover in personalised products we have to distinguish between a personalised product obtained from a current item, and one that is completely new (Lillrank et al. - 2006). Without falling in error, we can consider as "standard product" the personalised one obtaining from a mass produced article so its $\mathrm{NC}$ can be said "Mass-Product $\mathbf{N C}$ " $\left(\mathbf{N C}_{\mathbf{M P}}\right)$, while for the personalised products we will have the "Personalized Products $\mathrm{NC}^{\prime}$ ' $\left(\mathrm{NC}_{\mathrm{PP}}\right)$.

The $\mathrm{NC}_{\mathrm{PP}}$ have to deal in different way from the $\mathrm{NC}_{\mathrm{MP}}$ because they regard the production of a completely new product. When $\mathrm{NC}_{\mathrm{PP}}$ is generated, it will be set in a Corrective Action (CA), and this will generate an Ameliorative Action (AA) in order to avoid the $\mathrm{NC}_{\mathrm{PP}}$.

This wants to say that a $\mathrm{NC}_{\mathrm{PP}}$ related by an $\mathrm{AA}$ well executed has a low probability to happen (probability equal to 0 ) or if the AA has not been well planned or well executed the $\mathrm{NC}_{\mathrm{PP}}$ has a high probability to occur (probability 1). In order to standardize the number of $\mathrm{NC}_{\mathrm{PP}}$ we have assigned them a weight $(\sigma)$ equal to 0,5 .

The Quality Pointers conceived in this work address the task to measure the ability of the firm to realise a product perceived as "good" by the customer (Kackar - 1986).

With this aim we have defined a specific pointer (Mass Production Realization Efficiency) to be computed after processing given by (eq.2):

$\boldsymbol{I}_{\text {REM }} \%=1-\frac{\operatorname{Re}\left\{C \overline{N C}_{M P}\right\}}{N .{ }^{\circ} C N C_{M P \text { Tot }}}$ 
where:

${ }_{C N C_{M P T O t}}$ is the max possible number of CNC for mass-products

$\operatorname{Re}\{C \overline{C C}\}$ is the real floor of the average of CNC that we have on the same product in one day, week or, if the production is slow, every time the product is completed.

Moreover $\operatorname{Re}\left\{C \overline{C N C}_{M P}\right\}$ varies from 0 to $\mathrm{CNC}_{\text {MPTot }}$ so we have $\mathrm{CNC}_{\text {MPTot }}+1$ values of $\mathrm{I}_{\text {REM. }}$. The whole $\mathrm{I}_{\text {REM }}$ interval has now to be divided in "Classes" that measure the production efficiency.

The number of $\mathrm{NC}$ classes is function of the maximum number of possible $\mathrm{CNC}_{\mathrm{MP}}$ and in our approach has been calculated using the Sturges (Oppenheim, Shaffer, 1989) formula (eq.3), obtaining a real number that expresses the efficiency of the classes:

$N_{C}=1+3.3 \cdot \log _{10} \mathrm{~N}^{\circ} \mathrm{I}_{\text {REM }}$

Now we have front the main problem of our approach, i.e. to express these classes as an integer number and to lead us again to a prefixed number of efficiency cases, using the Uniform Quantization method (Gersho et al.- 1998).

As well known the Uniform Quantization is a correspondence that transforms the continuous set $\mathfrak{R}$ in the finite $\left\{\hat{x}_{1}, \hat{x}_{2}, \ldots \hat{x}_{M}\right\}$ one as follows:

$$
Q: x \in \mathfrak{R} \rightarrow \hat{x} \in\left\{\hat{x}_{1}, \hat{x}_{2}, \ldots \hat{x}_{M}\right\}
$$

Every input values inner to the interval ${\left(x_{i-1}, x_{i}\right)}$ - said quantization interval are represented with the only value $\hat{x}_{i}$ said restitution level, coinciding with the medium point of the quantization interval.

So in our case, fixing the max number of restitution levels $M$ to 4, we transform the continuous set $\mathrm{N}_{\mathrm{C}}$ with the value $\mathrm{N}_{\mathrm{QC}} \in\left\{\mathrm{N}_{\mathrm{QC} 1}, \mathrm{~N}_{\mathrm{QC} 2}, \mathrm{~N}_{\mathrm{QC} 3}\right.$, $\left.\mathrm{N}_{\mathrm{QC} 4}\right\}$. As shown in figure 1, all the input values of $\mathrm{N}_{\mathrm{C}}$ inner to interval $\left(\mathrm{N}_{\mathrm{Ci}-1}, \mathrm{~N}_{\mathrm{Ci}}\right)$, will be represented with the only one $\mathrm{N}_{\mathrm{QCi}}$, restitution level, that it will coincide with the maximum value of the quantization interval (and not with the medium point because of the necessity to represent $\mathrm{N}_{\mathrm{QC}}$ with an integer number). 


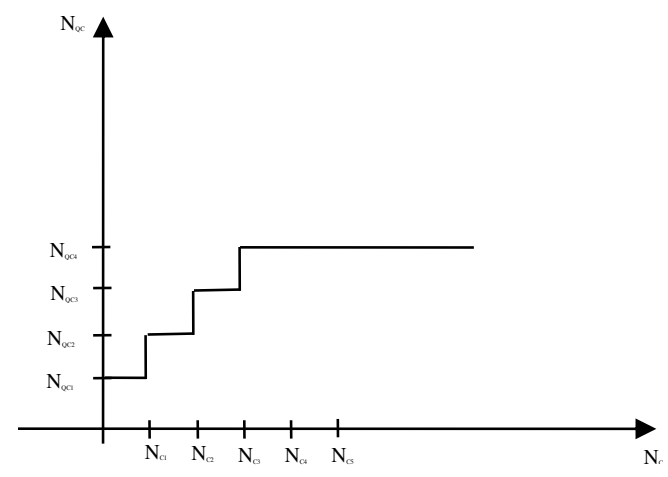

Figure 1: Uniform Quantization

That will be true only for the inner intervals and the inferior outside interval, while for that outside superior the minimal value of the interval will be chosen.

Not considering meaningful case of $\mathrm{N}^{\circ} \mathrm{I}_{\mathrm{REM}}=1$, we have proposed the quantization intervals as follows:

1. $\mathrm{N}_{\mathrm{C}}$ values inner the interval $[1,2]$ will be expressed with the restitution level $\mathrm{N}_{\mathrm{QC}}=2$ (max of external inferior interval $[1,2]$ );

2. $N_{C}$ values inner the interval $[2,3]$ will be expressed with the restitution level $\mathrm{N}_{\mathrm{QC}}=3$ (max of inner interval $\left.[2,3]\right)$;

3 . $N_{C}$ values inner the interval $[3,4]$ will be expressed with the restitution level $\mathrm{N}_{\mathrm{QC}}=4$ (max of inner interval $\left.[3,4]\right)$;

4. $N_{C}$ values inner the interval $[4, \infty]$ will be expressed with the restitution level $\mathrm{N}_{\mathrm{QC}}=4$ (minimum of outside superior interval [4, $\infty])$.

In this way we will have always a maximum number of classes equal to 4 , as shown in table 1 in which we can see as the restitution level $\mathrm{N}_{\mathrm{QC}}$ is always equal to 4 for a number of $\mathrm{I}_{\mathrm{REM}}$ (and so for $\mathrm{N}_{\mathrm{C}}$ ) greater than 4 . 


\begin{tabular}{|l|l|l|l|l|}
\hline $\boldsymbol{C N C}_{\text {MPTot }}$ & $\boldsymbol{N}^{\circ} \boldsymbol{I}_{\boldsymbol{R E M}}$ & $\boldsymbol{N}_{\boldsymbol{C}}$ & $\boldsymbol{N}_{Q \boldsymbol{C}}$ & $\Delta$ \\
\hline 9 & 10 & $4.40 \epsilon(4, \infty)$ & 4 & 25 \\
\hline 8 & 9 & $4.14 \epsilon(4, \infty)$ & 4 & 25 \\
\hline 7 & 8 & $3.98 \epsilon(3,4)$ & 4 & 25 \\
\hline 6 & 7 & $3.78 \epsilon(3,4)$ & 4 & 25 \\
\hline 5 & 6 & $3.56 \epsilon(3,4)$ & 4 & 25 \\
\hline 4 & 5 & $3.30 \epsilon(3,4)$ & 4 & 25 \\
\hline 3 & 4 & $2.98 \epsilon(2,3)$ & 3 & 33,33 \\
\hline 2 & 3 & $2.57 \epsilon(2,3)$ & 3 & 33,33 \\
\hline 1 & 2 & $1.99 \epsilon(1,2)$ & 2 & 50 \\
\hline
\end{tabular}

Table 1: Possible cases of $\mathrm{I}_{\mathrm{REM}}, \mathrm{N}_{\mathrm{C}}, \mathrm{N}_{\mathrm{QC}}$

The amplitude of a class $\Delta$ is calculable through the following relation (eq.5):

$\Delta=\frac{I_{R E M \max }-I_{R E M \min }}{N_{Q C}}$

where:

$\boldsymbol{I}_{\text {REMmax }}$ is the maximum value of Efficiency obtainable,

$\boldsymbol{I}_{\text {REMmin }}$ it is the minimum value of Efficiency obtainable.

In this way, supposing that the maximum number of critical $\mathrm{NC}$ found on a mass-product is equal to $7\left(\mathrm{CNC}_{\mathrm{MPT} T}=7\right)$ and that the number of measured $\mathrm{CNC}$ are $8[0,7]$, and so that $\mathrm{I}_{\mathrm{REM}}$ is equal to 8 and it has got eight values $\left(\mathrm{N}^{\circ} \mathrm{I}_{\mathrm{REM}}=8\right)$; the classes' number and the amplitude are:

$N_{C}=1+3.3 \cdot \log _{10} 8=4.14 \epsilon(4, \infty)$ so $\mathrm{N}_{\mathrm{QC}}=4 ; \Delta=25$.

Afterwards, in the case of $\mathrm{N}_{\mathrm{QC}}=4$ we have fixed the following four efficiency classes:

- Optimal Efficiency class (OE): it contains the values of $\mathrm{I}_{\mathrm{REM}}$ in the interval $] 75 \%, 100 \%$ ];

- High Medium Efficiency class (HME): it contains the values of I IEM in the interval $] 50 \%, 75 \%$ ];

- Low Medium Efficiency class (LME): it contains the values of $\mathrm{I}_{\mathrm{REM}}$ in the interval $] 25 \%, 50 \%$ ];

- Insufficient Efficiency class (IE): it contains the values of IREM in the interval $[0 \%, 25 \%]$. 
In the case of $\mathrm{N}_{\mathrm{QC}}=3$ we have defined ${ }^{1}$ :

- Optimal Efficiency class (OE): it contains the values of $\mathrm{I}_{\mathrm{REM}}$ in the interval ] $67 \%, 100 \%]$;

- Medium Efficiency class (ME): it contains the values of $\mathrm{I}_{\mathrm{REM}}$ in the interval ] $33 \%, 67 \%$ ];

- Insufficient Efficiency class (IE): it contains the values of IREM in the interval $[0 \%, 33 \%]$.

In the case of $\mathrm{N}_{\mathrm{QC}}=2$ we have defined:

- Optimal Efficiency class (OE): it contains the values of $\mathrm{I}_{\mathrm{REM}}$ in the interval ]50\%, 100\%];

- Insufficient Efficiency class (IE): it contains the values of I IRE in the interval $[0 \%, 50 \%]$.

Diagram 1 depicts the trend of $\mathrm{I}_{\mathrm{REM}}$ if $\mathrm{NC} \in[1 ; 5]$

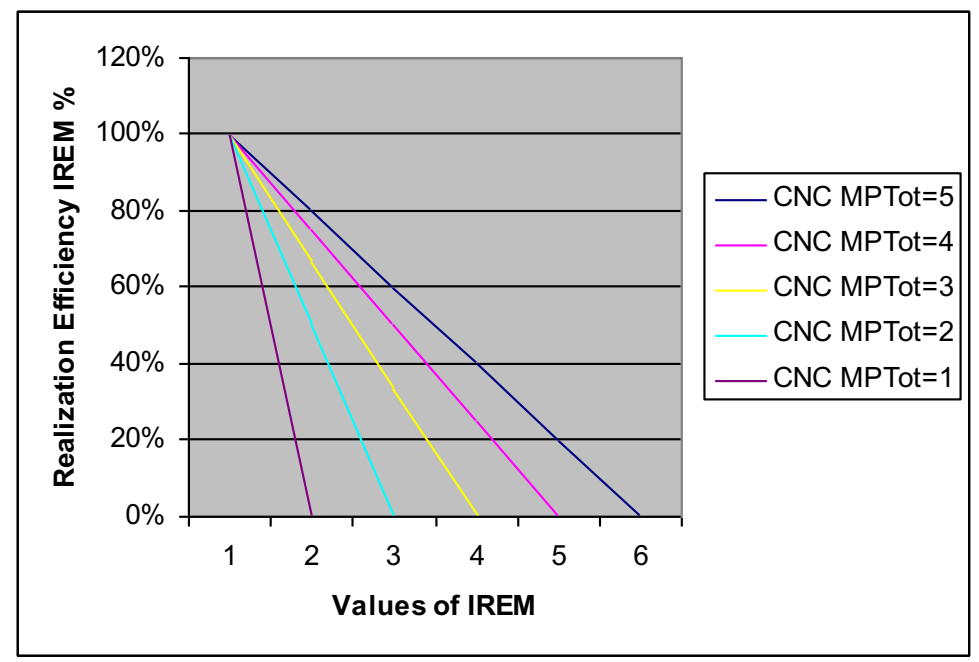

Figure 2: Trend of $I_{\text {REM }}$

Analysing Figure 2 we can notice that growing of the CNC totals there is a lessening of the slope of $I_{\text {REM }}$, because there is an increase of the number of the classes and so there is a greater difficulty in the attainment of the Optimal Efficiency.

For personalized products the $\mathrm{I}_{\mathrm{REP}}$ index has been defined as follows (eq. $6)$ :

1 we have created 2 classes of amplitude of $33 \mathrm{OE}$ and IE, and one of $34 \mathrm{ME}$ 
$\boldsymbol{I}_{\text {REP }} \%=1-\frac{\operatorname{Re}\left\{C \overline{N C_{P P}}\right\} * \sigma}{N .^{\circ} C N C_{P P T O t .}}(6)$

with the same notation of Equation (2).

Figure 3 depicts the trend of $I_{\text {REP }}$ with the same notations of the previous one.

Now we have to point out the both $\mathrm{I}_{\mathrm{REM}}$ and $\mathrm{I}_{\mathrm{REP}}$ gives the same indication (the ability of the firm to realise a product perceived as "good" by the customer); the difference between them is in the trend, explained in fig. 4. This, to mean that a specific non conformity perceived by a customer will have the same perception both in the mass and the personalised products.

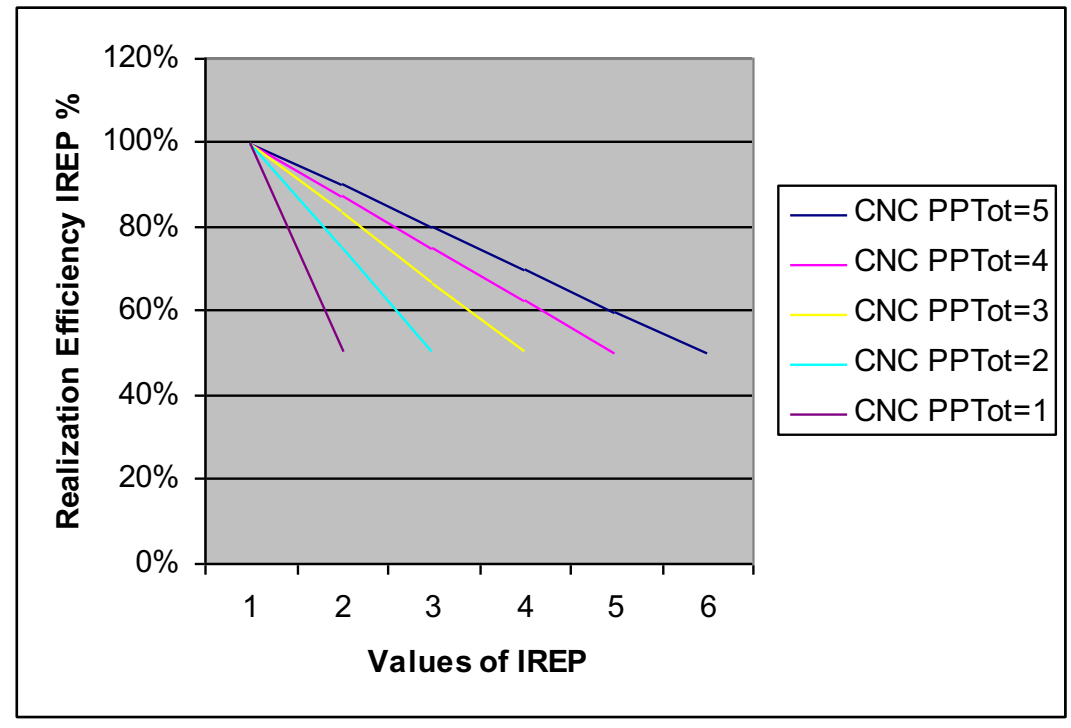

Figure 3: Trend of $I_{R E P}$

Fixed NC we have compared the two pointers (see figure 4); that points out that the slope of the IREP is greater because it is characterised by a greater difficulty to obtain a fixed quality level in case of personalised products: 


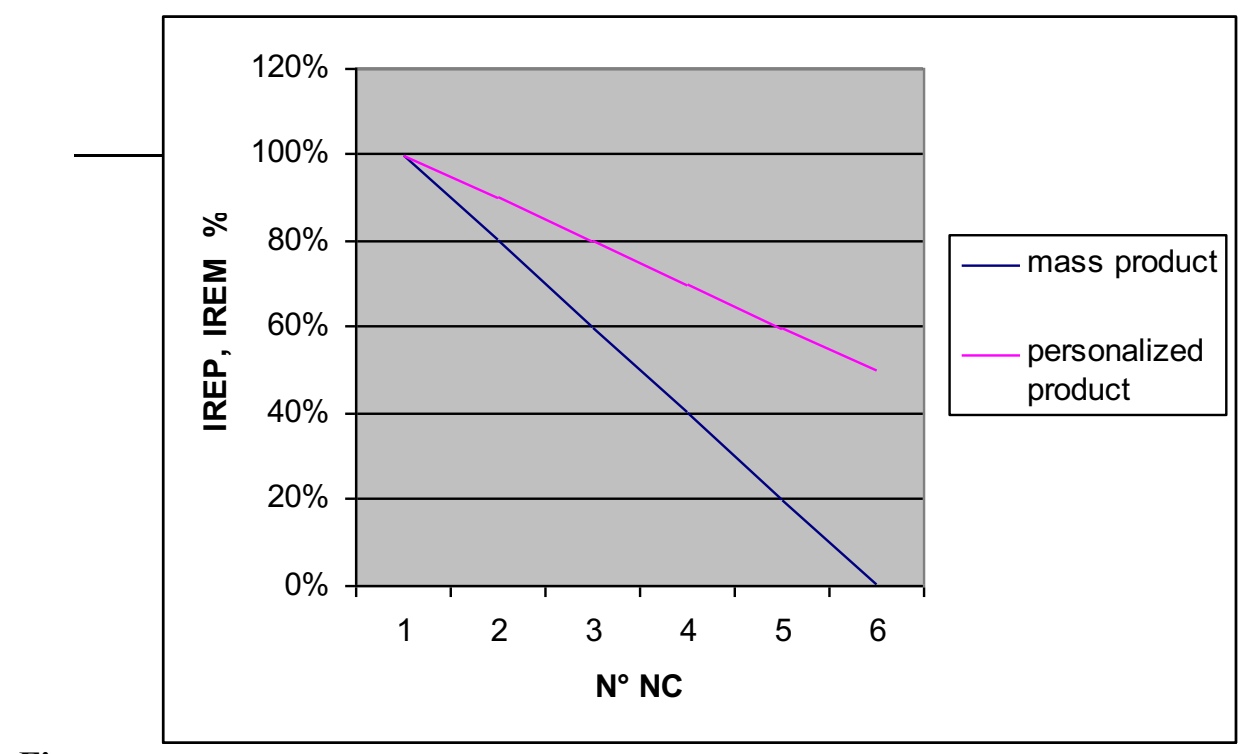

Figure

4: Comparison between $I_{R E M}$ and $I_{R E P}$

In this last case we have intentionally eliminated the low case, inserting the weight $\boldsymbol{\sigma}$ (it's useful to consider the restitution levels $\mathrm{M}=3$, in which we do not consider the case $\mathrm{N}^{\circ} \mathrm{I}_{\mathrm{REP}}=1$ ) and fixing the quantization interval as follows

1. All the $\mathrm{N}_{\mathrm{C}}$ values inner the interval $(1,2)$ will be expressed with the restitution level $\mathrm{N}_{\mathrm{QC} 1}=2$ (max of external inferior interval $(1,2)$ );

2. All the $\mathrm{N}_{\mathrm{C}}$ values inner the interval $(2,3)$ will be expressed with the restitution level $\mathrm{N}_{\mathrm{QC} 2}=3$ (max of inner interval $(2,3)$ );

3 . All the $N_{C}$ values inner the interval $(3, \infty)$ will be expressed with the restitution level $\mathrm{N}_{\mathrm{QC} 3}=3$ (minimum of outside superior interval (3, $\infty)$ ).

In case of three classes $\left(\mathrm{N}_{\mathrm{QC}}=3\right)$ we define ${ }^{2}$ :

- Optimal Efficiency class (OE): it contains the values of $I_{\text {REP }}$ in the interval ] $83 \%, 100 \%]$;

- Medium Efficiency class (ME): it contains the values of $I_{\text {REP }}$ in the interval ]67\%, 83\%];

- Insufficient Efficiency class (IE): it contains the values of $I_{\text {REP }}$ in the interval ]50\%, 67\%].

In case of two classes $\left(\mathrm{N}_{\mathrm{QC}}=2\right)$ we define:

- Optimal Efficiency class (OE): it contains the values of $\mathrm{I}_{\mathrm{REP}}$ in the interval ] $75 \%, 100 \%]$;

- Insufficient Efficiency class (IE): it contains the values of $I_{\mathrm{REP}}$ in the interval $[50 \%, 75 \%]$.

The I $I_{\text {REM }}$ and $I_{\text {REP }}$ values now have to be compared with the goals fixed in the factory's Quality Plan [10]. If we represent on XY plane the objective

2 we have created 2 classes of amplitude of $17 \mathrm{OE}$ and ME, and one of $16 \mathrm{IE}$ 
values of reference ( $Y$ axis), and the values of the indicators $I_{\text {REM }}$ and $I_{R E P}$ (X axis), we will have (figure 5):

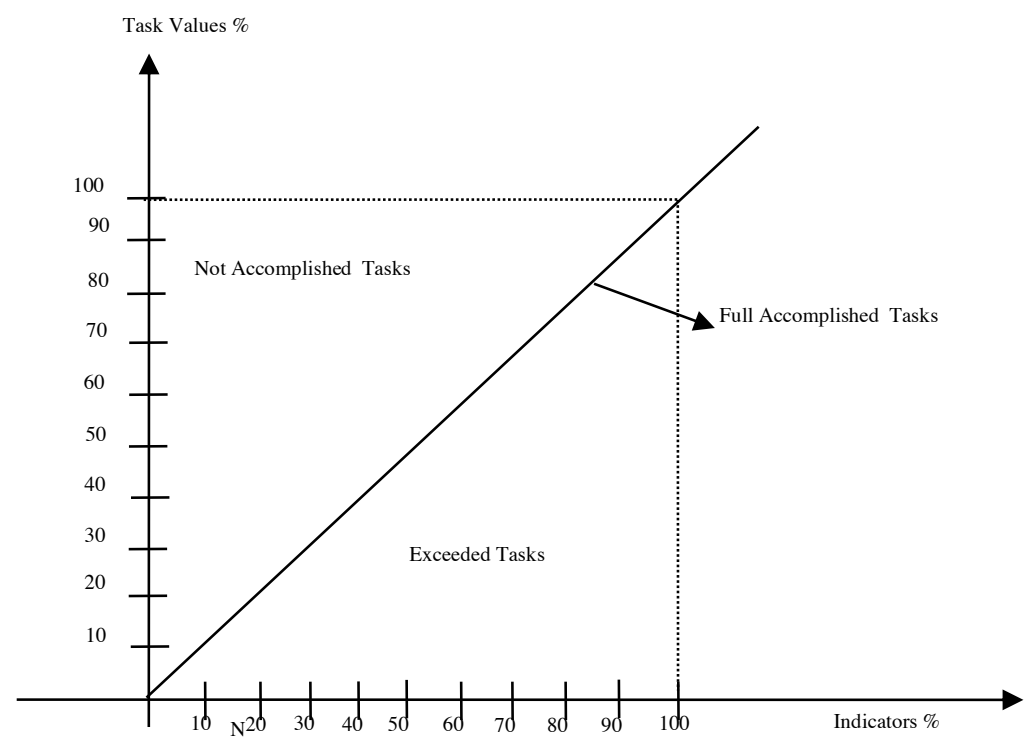

Figure 5: Quality Task Plane - QTP

The points over the bisector represent the Not Accomplished Tasks case, while the points on the bisector and those under the bisector indicate respectively the full accomplished and the overcoming prefixed tasks. In these two cases the factory should not set any particular strategy because it has accomplished the prefixed Realization Efficiency.

As an operative example let's suppose that the factory has decided to accomplish a Realization Efficiency task equal to $50 \%$. If the measured efficiency is equal to $60 \%$, under bisector case, it seems that the factory doesn't set any procedure because it has exceeded the prefixed task.

But $60 \%$ value could belongs to an Optimal Efficiency or Medium Efficiency class so the management has to project a plan to accomplish the Medium or Optimal Efficiency (Dale et al - 1998, Lillrank et al. 2006).

In this way using our approach, they are able to identify an improvement policy because they can fix a priority with the following intervention levels:

- High Priority (H.P.): urgent actions;

- Medium Priority (M.P.): short time actions;

- Low Priority (L.P.): medium time actions;

- Null Priority (N.P.): long time actions. 
The priorities are assigned through a specific instrument called "Actions Priority Table" (Table 2) which relates the points of the Quality Task Plane (QTP) shown in fig. 5 to the Efficiency Classes previously defined. Entering on QTP with the value of $I_{\text {REM }}$ or $I_{\text {REP }}$, we can compare it with the Realisation Efficiency set by the firm. With this comparison the following Action Priority Table gives the type of action that has to be taken for the analysed product.

Referring to fig. 5, in Tab. 2 the $\boldsymbol{O B P}$ are the Over Bisector Points, BP are the Bisector Points, while the UBP are the Upper Bisector Points

\begin{tabular}{|l|l|l|l|l|l|}
\cline { 2 - 6 } \multicolumn{1}{c|}{} & \multicolumn{5}{c|}{ EFFICENCY CLASSES } \\
\hline POINTS & $I E$ & $L M E$ & $M E$ & $H M E$ & $O E$ \\
\hline$O B P$ & H.P. & H.P. & M.P. & L.P. & L.P. \\
\hline$B P$ & H.P. & H.P. & M.P. & L.P. & N.P. \\
\hline$U B P$ & H.P. & M.P. & M.P. & M.P. & N.P. \\
\hline
\end{tabular}

Table 2: Actions Priority Table

In the previous table the priority level is independent from the number of classes and the H.P. level is obtained for IE and LME Classes - where a strong action is demanded in a short interval of time - while M.P. and L.P. levels are necessary for ME, HME and OE classes; N.P. appears only in OE class.

Figure 6 gives the workflow of the entire approach:

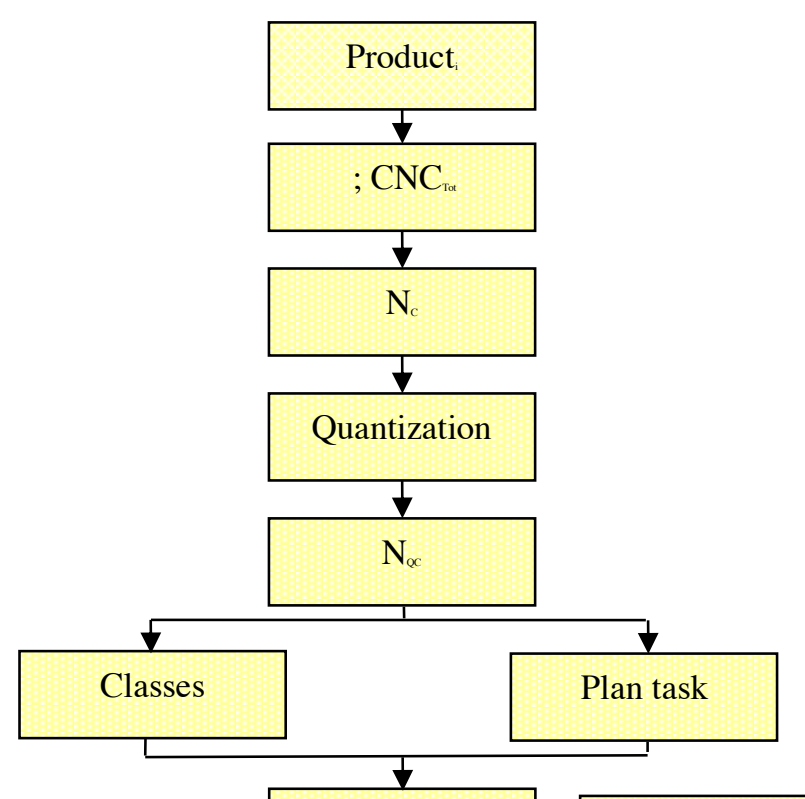




\section{Figure 6: Flow diagram of the approach}

\section{The industrial case study}

The described approach has been implemented in a factory producing recreational vehicles like Motorcaravans, Motorhomes and Roulottes. For every each model there are several mass-products and different personalization can be choosen.

This factory produces a moulding Monocoque body made of plastic reinforced by incorporated fibreglass inner a stamp. Firm's quality policy wants to achieve a good production level of the Monocoque body (i.e. Realization Efficiency equal to $70 \%$ ).

As first step we have analysed the NC of the Monocoque body in the production process, so we have:

- impurities;

- imperfections;

- superficial cracks;

- air bubbles;

- irregulars weldings;

- shape errors.

Afterwards the following $\mathrm{CNC}$ have been defined:

- superficial cracks;

- air bubbles;

- irregulars weldings;

- shape errors

and we have fixed four typologies of $\mathrm{CNC}\left(\mathrm{N}^{\circ} \mathrm{CNC}_{\mathrm{MPTot}}=4\right)$.

The number of possible value of $I_{\text {REM }}$ is equal to 5 , so we have a number of classes $\mathrm{N}_{\mathrm{C}}=1+3.3 \log _{10} 5=3.30 \in(3,4)$; after quantization, we have $\mathrm{N}_{\mathrm{QC}}=4$ with the following classes: IE, LME, HME, OE.

We have analysed the production during three months (12 Monocoque bodies have been produced) obtaining the following results (Table 3 ). 


\begin{tabular}{|l|l|l|}
\hline CNC Tipology & CNC Measured & \% CNC \\
\hline air bubbles & 10 & 83 \\
\hline superficial cracks & 6 & 50 \\
\hline shape errors & 5 & 42 \\
\hline irregulars weldings & 8 & 67 \\
\hline
\end{tabular}

Table 3: CNC Monocoque Bodies

Analysing table 3 we can notice that there is a high percentage of air bubbles $(83 \%)$ and irregulars weldings $(67 \%)$ and a $60 \%$ of total $\mathrm{CNC}$, 29 on 48 possible ones - infact for every Monocoque body we can have only 4 typologies of $\mathrm{CNC}$ and for 12 Monocoque Bodies we have a total of $48 \mathrm{CNC}$. With these values we have calculated the mean value of $\mathrm{CNC}$ and $\mathrm{I}_{\text {REM }}$, obtaining:

$$
\operatorname{Re}\{C N C\}=\operatorname{Re}\{2,41\}=2 ; \quad I_{R E M}=1-\frac{2}{4}=50 \%
$$

The Realization Efficiency Indicator, with a value of 50\%, set the Massproduction of Monocoque bodies in the LME class (] 25\%, 50\%]) so we have obtained an Over Bisector Point that represents, with LME class, a H.P. level.

The factory will have to increase the Efficiency Realization of $24 \%$ to achieve the Task Value of the $70 \%$.

We set $\mathrm{I}_{\text {REM }}=70 \%$ so we must have a $\overline{C N C}_{T}=1.2$ (instead of 2); but the relation of $\mathrm{CNC}$ gives the following result

$\overline{C N C}_{T}=\frac{\sum_{i=1}^{P} N^{\circ} C N C_{i}}{P} \Rightarrow \sum_{i=1}^{P} N^{\circ} C N C_{i}=P \cdot \overline{C N C}_{T}=12 \cdot 1,2 \approx 19$

where P represent the number of Monocoque body analysed, while

$\overline{C N C}_{T}=19$ corresponds to $30 \%$ of total CNC.

With these experimental results to achieve the Task Value of Efficiency of $70 \%$, the firm must obtain a decreasing of the total number of $\mathrm{CNC}$ from $60 \%$ to $30 \%$.

The strategy adopted by the management to obtain this task has been to distribute the difference of $30 \%$, to decrease the number of $\mathrm{CNC}$ of the air bubbles from $75 \%$ to $55 \%$, and the number of $\mathrm{CNC}$ of irregular welding from $67 \%$ to $57 \%$.

\section{Conclusions}

This work addresses the problem of pointing out and interpreting the trend of a Quality Management System, giving to the firm management 
the possibility to decide the specific priorities of actions to be taken to improve product quality. The different level of priority, with respect to quality tasks, has been defined with a specific dedicated approach that uses specific indexes ( $\mathrm{I}_{\text {REM }}$ and $\mathrm{I}_{\text {REP }}$ ) which differentiate the cases of personalized products or standard ones. The five efficiency classes have been exploited through the Uniform Quantization method. The approach has been organized in a structured procedure that can be implemented in industrial context characterized by small lots production and/or high personalised products. The approach is completely independent by the production process; moreover it is also sensible to those firms that do a high personalization of their products or produce with a single-item commitments, considering with less severity the $\mathrm{CNC}$ of a personalized product.

Future studies could optimize the intersection on Task Plane between the task values and indicators, giving a careful intervention method, after the indication of the level priority, which could be described in detail in the specific production context. Another evolution of this work can be intended toward the direction of expressing the $\mathrm{NC}$ classes with the method of Fuzzy Sets; especially with regard to $\mathrm{CNC}_{\mathrm{PP}}$, their trend can be expressed by a fuzzy number function of the Ameliorative Actions taken, researching a critical limit related to the introduction of specific corrective measures. This kind of approach could fit better those production/services context in which $\mathrm{NC}$ and $\mathrm{CNC}$ cannot often be expressed by a specific defectiveness of the product and/or qualitative data are characterised by inconsistency.

Another structured approach for correctly defining this matter may be the object of a further development of this work.

\section{References}

1. Paul Lillrank, Jaakko Kujala, 2006, Managing common and specific causes of quality problems in project-based organisations, International Journal of Productivity and Quality Management - Vol. 1, No. 1, pp. $56-68$;

2. Alok Mishra, Deepti Mishra, 2006, Software quality assurance models in small and medium organisations: a comparison, International Journal of Information Technology and Management, Vol. 5, No.1, pp. $4-20$;

3. John S. Oakland, Steve J. Tanner, 2006, Quality management in the 21 st century - implementing successful change, International Journal of Productivity and Quality Management, Vol. 1, No.1/2, pp. 69 - 87;

4. Richard A. Reid, 2006, Productivity and quality improvement: an implementation framework - International Journal of Productivity and Quality Management, Vol. 1, No.1/2, pp. 26-36; 
5. Rodney McAdam, Catherine McEnroe, 2004, A framework for measuring quality improvement in Irish supplier SMEs: a multiple case analysis, International Journal of Business Performance Management, Vol. 6, No.2, pp. 171 - 188;

6. Tritos Laosirihongthong, Himangshu Paul, 2004, Competitive manufacturing strategy: an application of quality management practices to advanced manufacturing technology implementation, International Journal of Business Performance Management, Vol. 6, No.3/4, pp. 262 - 286;

7. C. Calligaro, 2002, Misurare il Miglioramento - Indicatori di Qualità da Azioni Correttive e Preventive, National Journal De Qualitate, Vol.

$\mathrm{XI}, \mathrm{N}^{\circ} 7, \mathrm{pp} .30-35$;

8. Louis E. Solis, S. Subba Rao, T.S. Ragu-Nathan, 2001, The best quality management practices in small and medium enterprises: an international study, International Journal of Manufacturing Technology and Management, Vol. 3, No.4/5, pp. 416-443;

9. Barrie Dale, Ruth Boaden, Mark Wilcox, Ruth McQuater, 1998, The use of quality management techniques and tools: an examination of some key issues, International Journal of Technology Management Vol. 16, No.4/5/6, pp. 305-325;

10. Amrik S. Sohal, Edmund Lu, 1998, Requirements for successful implementation of total quality management, International Journal of Technology Management, Vol. 16, No.4/5/6 pp. 336-357;

11. R.M. Gray and D.L. Neuhoff, 1998, Quantization, IEEE Transactions on Information Theory, Vol. 44, pp. 2325--2384,

12. A.V. Oppenheim, D. Shaffer, 1989, Discrete Time Signal Processing, New Jersey, Prentice Hall;

13. Gerho, A., Gray, R.M, 1992, Vector quantization and signal compression, Vol. 1, Kluwer Academic, Boston;

14. Hotard, D. G., 1988, Quality and Productivity: An Examination of Some Relationships, Vol. 4, pp. 259-266, Engineering Management International;

15. Kackar, R. N., 1986, Taguchi's Quality Philosophy: Analysis and Comment, Quality Progress, pp. 21-29; 